\title{
Information Infrastructures for eHealth
}

\author{
Margunn Aanestad, Miria Grisot, Ole Hanseth, \\ and Polyxeni Vassilakopoulou
}

\section{$2.1 \quad$ Introduction}

This chapter provides an introductory overview of healthcare information systems, followed by a more detailed discussion of e-prescription and governmental patient-oriented platforms. We use the umbrella term "eHealth" (also written e-health) that encompasses all health-related digital information systems including clinical, administrative, and research-oriented ones. Specifically, we adopt the eHealth definition introduced by the World Health Organization (WHO). According to this definition, eHealth is "the use of information and communication technologies (ICT) for health; examples include treating patients, conducting research, educating the health workforce, tracking diseases and monitoring public health" (World Health Organisation 2016b). Similarly, the European Commission defines eHealth as: "the use of modern information and communication technologies to meet needs of citizens, patients, healthcare professionals, healthcare providers, as well as policy makers" (European Commission 2003). eHealth is considered pivotal for improving the quality and efficiency of healthcare (Hillestad et al. 2005; Kellermann and Jones 2013), for improving the patient experience of care, and for the eventual revolutionization of healthcare (Drucker 2007).

\footnotetext{
M. Aanestad ( $\square)$

University of Oslo \& UIT The Arctic University of Norway,

P.O. Box 1080 Blindern, N-0316 Oslo, Norway

e-mail: margunn@ifi.uio.no

M. Grisot • O. Hanseth

University of Oslo, P.O. Box 1080 Blindern, N-0316 Oslo, Norway

e-mail: miriag@ifi.uio.no; oleha@ifi.uio.no

P. Vassilakopoulou

University of Agder \& University of Oslo, P.O. Box 422, N-4604 Kristiansand, Norway

e-mail: polyxenv@uia.no
} 
Strong expectations linked to eHealth are present in policy and advisory documents prepared around the globe. For instance, the introductory passage of a report by the Institute of Medicine of the National Academies (US) states: "Health and health care are going digital. As multiple intersecting platforms evolve to form a novel operational foundation for health and health care the stage is set for fundamental and unprecedented transformation." (Institute of Medicine 2011). In Europe, eHealth has been a major component of the European Commission's eEurope action plan which was endorsed at the Feira European Council in June 2000. In 2004, the Commission also set in place an eHealth map to develop targeted policy initiatives aimed at fostering widespread adoption of eHealth technologies across the EU (eHealth Action Plan). The latest eHealth Action Plan for 2012-2020 states that the promise of eHealth "remains largely unfulfilled" and the vision of a unified, interoperable eHealth Infrastructure in Europe is still not realised. Although the potential of eHealth is being discussed globally since the 1990s it remains a work in progress.

Countries around Europe have already experienced notable successes and some highly publicised costly delays and failures. These have brought attention to the complexity of dealing with a multiplicity of involved parties with diverging interests and agendas, existing fragmented systems' landscape, rapid technological advancements and regulative perplexities. In most European countries, healthcare is predominantly public and public agencies have a central role for stimulating and orchestrating eHealth efforts. In many countries, the driving force for ICT in health care has been the trend toward a better coordination of care (Winter et al. 2011). This means a change of focus for eHealth from self-contained processes within single healthcare institutions to overall care processes spreading across institutional boundaries.

The remainder of this chapter is structured as follows. In the next section we give an overview of the eHealth landscape. Then, in Sects. 2.3 and 2.4 we focus on the two types of infrastructures examined in this book: e-prescription and governmental patient-oriented platforms. Finally, Sect. 2.4 concludes the chapter with a discussion on the transformative potential of the two types of eHealth infrastructures.

\subsection{The eHealth Landscape}

To provide the necessary background for the reader, we initially describe information systems that support healthcare-related work within specific organizational settings (e.g. laboratories, medical imaging departments, general practitioner offices). Next, we move beyond these systems, and we present systems that have more generic character and are common enabling components for eHealth.

\subsubsection{Core Information Systems in Healthcare Organizations}

There is a multitude of systems that support healthcare provision ranging from more generic systems to the ones that offer specialised functionalities for specific domains. Among the specialized, for example there are Picture archiving and 
communication systems (PACS) which support storage, retrieval, management, distribution and presentation of medical images, and RIS (Radiology Information Systems) which support patient administration, referrals, reports, and work lists for the medical imaging labs. Computerized physician order entry (CPOE), medication management and vital signs monitoring systems are other examples of special-purpose systems. Of more generic use are Patient Administrative Systems (PAS), also called Admission-Discharge-Transfer (ADT) systems that support registration, scheduling and logistics and Electronic Health Record systems (EHRs). EHRs play a central role in health institutions. An EHR is envisioned as a "repository of information regarding the health of a subject of care in computer processable form, stored and transmitted securely, and accessible by multiple authorised users. It has a commonly agreed logical information model which is independent of EHR systems. Its primary purpose is the support of continuing, efficient and quality integrated health care and it contains information which is retrospective, concurrent and prospective" (ISO/TR 20514 2005). EHRs organize information related to specific patients and may cover several encounters and episodes of care, possibly from birth to death. The information within an EHR may be generated during patient encounters (e.g. diagnoses, lab results, radiology scan reports, etc.) and may also come from the patients (e.g. off-theshelf medicine, home measurements etc.). This information may be contained in multiple (discrete or interconnected) systems and repositories, each of which will hold and manage specific types of data (Winter et al. 2011). In addition to the systems that directly support healthcare provision, there is also a multitude of systems that support management functions (e.g. systems for management reporting, systems for reimbursement handling, etc.) and research activities (e.g. advanced computational tools for genetic data). There are also systems that support generic, but indispensable services such as user authentication and authorisation services.

\subsubsection{Information Systems Beyond the Healthcare Organization}

Beyond the spectrum of systems supporting work within the boundaries of a specific healthcare organization, there is also a class of systems and technological capabilities that are more generic, over-arching and serve as common enabling components for a wider eHealth infrastructure. Inter-organizational networks and messaging services for instance, facilitate information flow between organizations (e.g. message exchange between different healthcare providers) and across different levels within the healthcare system (e.g. reporting activities to health authorities and clinical information to health registries). These require the existence of shared infrastructural services like address registries, broadband networks and security infrastructures. In addition, information needs to be shared along a patient's trajectory if it involves diagnosis and treatment in multiple different localities and organizations. To enable easy access to relevant information about a patient, governments have sought to build 
cross-cutting systems such as e-prescription systems and shared EHRs (often in the form of summary or emergency care record systems). Standards, both interoperability standards and terminology and nomenclature standards are crucial components in facilitating eHealth infrastructures that go beyond organizational boundaries.

Such inter-organizational eHealth information infrastructures are important for multiple users in different organizational settings: clinical and administrative healthcare professionals, health researchers, public health authorities, health insurance companies and various other involved actors. Furthermore, a continuously growing number of eHealth systems are covering the interaction between patients and healthcare providers, or peer-to-peer communication between patients or health professionals. In this book, we explore infrastructures for e-prescription and patient-oriented platforms. Both of them are inter-organizational and have been a strategic priority for several countries recently.

\subsection{E-Prescription}

E-prescription solutions support the electronic flow of information related to prescribed medications. Most European countries have taken steps for implementing e-prescription solutions while the aim of the European Union is to have a cross-border electronic system which will enable patients to retrieve electronic prescriptions anywhere in Europe (World Health Organisation 2016a). Nevertheless, there are different degrees of maturity and coverage of e-prescription solutions in the different European countries. In some countries, e-prescribing is used routinely while in other countries there are only some early-stage initiatives.

\subsubsection{Prescriptions and e-Prescribing}

Modern medicine relies heavily on the use of medication. The production, distribution and use of medication is regulated by longstanding institutions. Over-thecounter medication can be purchased freely and used by anybody without medical supervision. If a medication is not available over-the-counter it can only be dispensed when a prescription is provided, to ensure that its use happens within a care scheme approved by a healthcare professional. National regulations govern who can issue a prescription. In general, doctors have the broadest prescriptive authority and are the main prescribers everywhere in the world. Additionally, other healthcare professionals (for instance: dentists, midwives, pharmacists) may also have the right to prescribe medications related to their area of practice; this varies from country to country.

A prescription may be handwritten on a clean sheet of paper or on pre-printed forms, or typed and printed, or transmitted electronically to pharmacies for dispensing. The content of a prescription includes information about both the patient and 


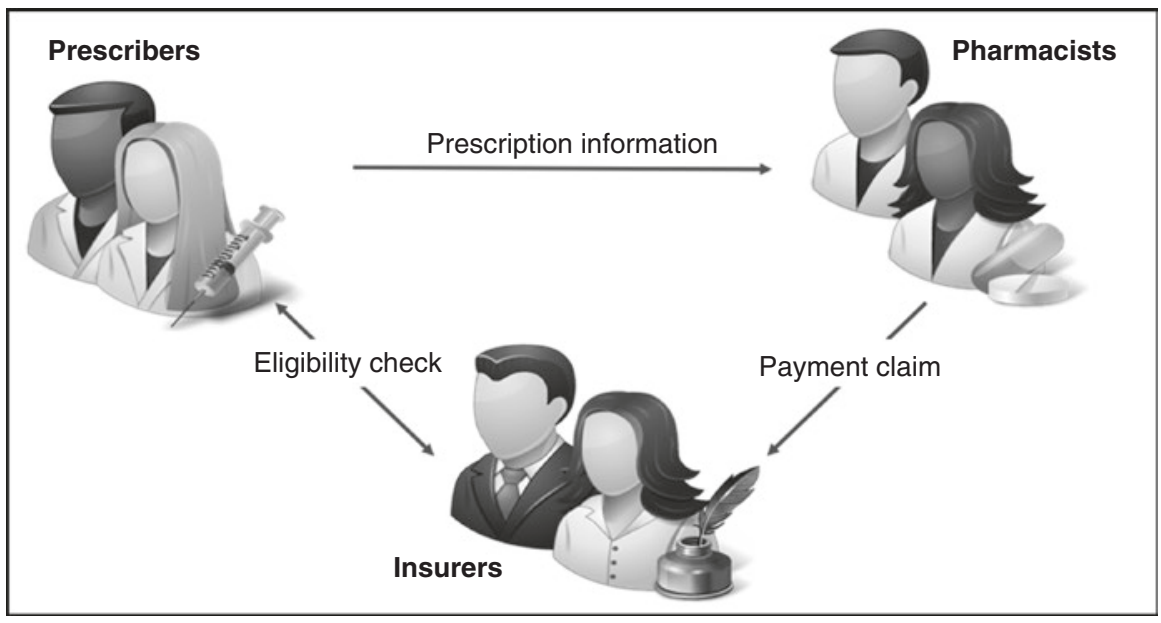

Fig. 2.1 Information flows between prescribers, pharmacists and insurers

the prescriber, the medication specifications (type, quantity) and directions for the patient to follow. Prescribed medication can be partially or fully reimbursed by healthcare insurers (public or private), hence, prescription information is also needed by insurers.

Health authorities around the world support the adoption of electronic prescription systems (e-prescription). E-prescription solutions capture and circulate prescription information between prescribers, pharmacies and insurers that handle related payments (Fig. 2.1) expediting flows and eliminating legibility issues (frequently faced when using handwritten prescriptions). Such solutions can support aims for cost containment, enhancement of patient safety, control over doctors' prescription patterns and process quality assurance. Overall, putting e-prescription in place entails working with multiple and diverse sociotechnical components, finding ways to link and organise them (Rodon and Silva 2015).

Beyond the traditional use of prescriptions in primary care, in hospital settings and in nursing homes, prescription information is needed by nurses that are administering medications. Furthermore, prescription information may be collected and processed by health policy institutions for planning and monitoring purposes. Overall, medication prescriptions and dispense data are monitored for various reasons, for instance, public health authorities may monitor and regulate the use of antibiotics, may monitor and exercise health control over the use of reimbursable drugs, may monitor and supervise imports and distribution. Therefore, most countries have an information infrastructure around the medical prescription. These information infrastructures can be paper-based or digital or in hybrid form and typically link multiple Health Record Systems, Pharmacy Systems, Drug Registries and Health Insurance Systems (electronic or not). 


\subsubsection{Key Parts of e-Prescription and Variations}

It is common to distinguish between three parts of e-prescription infrastructures:

eCapture: support in producing notes for prescribed medication. This can be a simple tool for registering electronically medication information (ensuring quick transmission and elimination of illegibility issues) or more elaborate arrangements that include decision support functionalities such as automatic checking of drug interactions (based on other information from the patient record), automatic retrieval of commercially available drugs and package sizes, support for the selection of drugs with the use of protocols based on the diagnosis descriptions.

eTransfer: transfer of the prescription information. Both electronically generated prescriptions and paper prescriptions filled by hand and scanned can be transferred digitally. Various models are adopted, for instance, the prescription can go from the prescriber to a specific pharmacy, or it can be deposited to a central repository accessible by all pharmacies (allowing the patient to choose where to go at a later stage). With electronic transfers the information flows can be expedited and also, it is possible to better control the duration of prescription's validity (for instance, the message or the information content can expire after a set date). Furthermore, the electronic transfer of prescriptions can allow secondary uses of the data (e.g. facilitating the checking and payment of pharmacy claims and the accumulation of information to support quality healthcare and effective cost management).

eDispensing: support in producing records of the actual medication dispensing. This can be a simple note on the date and place of dispensing or can include complete medication packaging information allowing full traceability and control of drugs.

The coverage of e-prescription projects varies in terms of:

- Actors: the e-prescription infrastructure must cover at least pharmacies and prescribers. In many cases e-prescription is covering only key prescribers (e.g. General Practitioners in primary care). In other cases it includes also hospitals, or even, other prescribers depending on national regulations (e.g. dentists, midwives, pharmacists). Furthermore, most e-prescription systems cover also information flows to insurers.

- Functionality: basic or advanced support for eCapture (e.g. might include decision support for prescribers), eTransmission (can be fully digital or quasi-digital e.g. paper with barcodes), eDispensing (registration of extended or limited information upon dispensing). Additional functionality may include facilities for patients to trigger prescription refills, full integration with Electronic Health Record Systems (EHRs), repository management facilities.

- Access: rules for data access can vary depending on national regulations and on designers' choices. Actors that can access personalised medication lists may 
include prescribing healthcare professionals, other healthcare professionals that provide services to the patient, pharmacists, public authorities, private insurers and patients.

There are variations among countries with different health systems. Variations relate to: what constitutes prescriptions drugs, who can issue a prescription, what is the minimum required content of a prescription, who can dispense a prescription, how medications are reimbursed. There are also legal differences: is electronic transmission of prescriptions legal? Are digital signatures accepted? Does the patient need to consent? Should the patient be able to request a paper copy? Is counselling compulsory before prescriptions are written? In Europe, each country has some particularities, for example: in UK there is some authority transferred to community pharmacies, in Norway nurses can prescribe some drugs (e.g. contraceptives), in Greece and Italy there is control over the physical medication packages that have unique identification numbers. Also, there are differences on insurance schemes for medication reimbursement. For example, in some countries (e.g. Norway) public insurance is unified while in other countries (e.g. Germany and Greece), there are multiple insurance institutions or social security funds.

\subsubsection{Drivers for e-Prescription Projects}

Expenses for medications contribute significantly to total healthcare expenditures. The expenditure on medications as a share of overall health expenditure varies throughout Europe ranging from $6 \%$ (Denmark, Norway) up to $29 \%$ (Greece), furthermore, the public share of this medication expenditure can range from less than $50 \%$ (Denmark, Finland) up to around $70 \%$ (Germany, Greece) (OECD 2013; World Health Organisation 2014). Therefore, it is seen as critical for governmental authorities to monitor and control prescriptions not only for ensuring healthcare quality but also for reasons of cost control. The expectations for better cost control fuelled the interest for e-prescription systems in European countries during the past decade. Sixteen of the member states of the EU included e-Prescription in their national strategies or eHealth implementation plans already in 2006; in 2011 this number was raised to 22 (Stroetmann et al. 2012). Still, in 2011, only Denmark, Estonia, Iceland and Sweden had in place a full, national e-Prescription solution while at the same time, there were partial implementations in the UK and the Netherlands, regional implementations in Spain, and several initiatives including pilots in Portugal, Czech Republic, Finland, Italy, Norway, Greece and Poland (Kierkegaard 2013; Stroetmann et al. 2011).

With the introduction of e-prescription the collaboration between physicians and pharmacists is mediated by technologies. E-prescription reduces the risks associated with traditional prescription-writing, and has the potential of bringing different benefits to different stakeholders, especially if implemented at scale (Cornford et al. 2014). At the same time, the inscription of rules to the system can be a powerful 
control mechanism for prescribers and dispensing pharmacists. In this respect, e-prescription has a dual role: it is not only a tool introduced to everyday work to improve healthcare delivery but also, a governance mechanism for regulating, controlling and monitoring a large array of dispersed temporally and geographically professional tasks (Vassilakopoulou et al. 2012).

In the chapters included in the e-prescription section of this book we present the experiences of different European countries that implemented e-prescription during the past decade. The different cases illustrate different strategies for linking preexisting infrastructural arrangements (the installed base) to new technological solutions and for extending and renewing the overall prescription related infrastructures. The cases are linked to each country context, the specific characteristics of health systems, the technological maturity of the healthcare environment and the different institutional actors. The cross-examination of the cases can bring a number of insights about different implementation approaches and overall, about the dynamics of infrastructural evolution.

\subsection{E-Services for Patients and Citizens}

The development of patient-oriented eHealth services is recent. Traditionally, healthcare information systems were developed for clinical and administrative use of health personnel in the context of healthcare organizations. However, recently several countries have initiated projects for establishing patient- or citizen-oriented eHealth solutions and infrastructures. Overall, the aim of these initiatives is to put in place secure and reliable technologies allowing patients to access general and personalised health information and providing electronic services for communication, self-management, and administrative tasks.

\subsubsection{Patient-Oriented eHealth Services}

Patient-oriented eHealth services are diverse (Fig. 2.2). Some services are mainly information-oriented. For instance, many governmental eHealth websites, but also hospital websites provide citizens with updated and quality-assured information about symptoms and treatment options. These services respond to the increasing interest for using the Internet as a source for health information, and to the problem of the variable quality of information available. Other services are set up to offer access to personal health data that healthcare institutions have registered about individuals, e.g. in the patient record systems, laboratory and imaging systems etc. To support the collation and use of personal health data, various specialised solutions for Personal Health Records (PHR) have been developed. PHRs are in some cases standalone patientcontrolled solutions, while in other cases as "tethered" to institutional EHRs. 


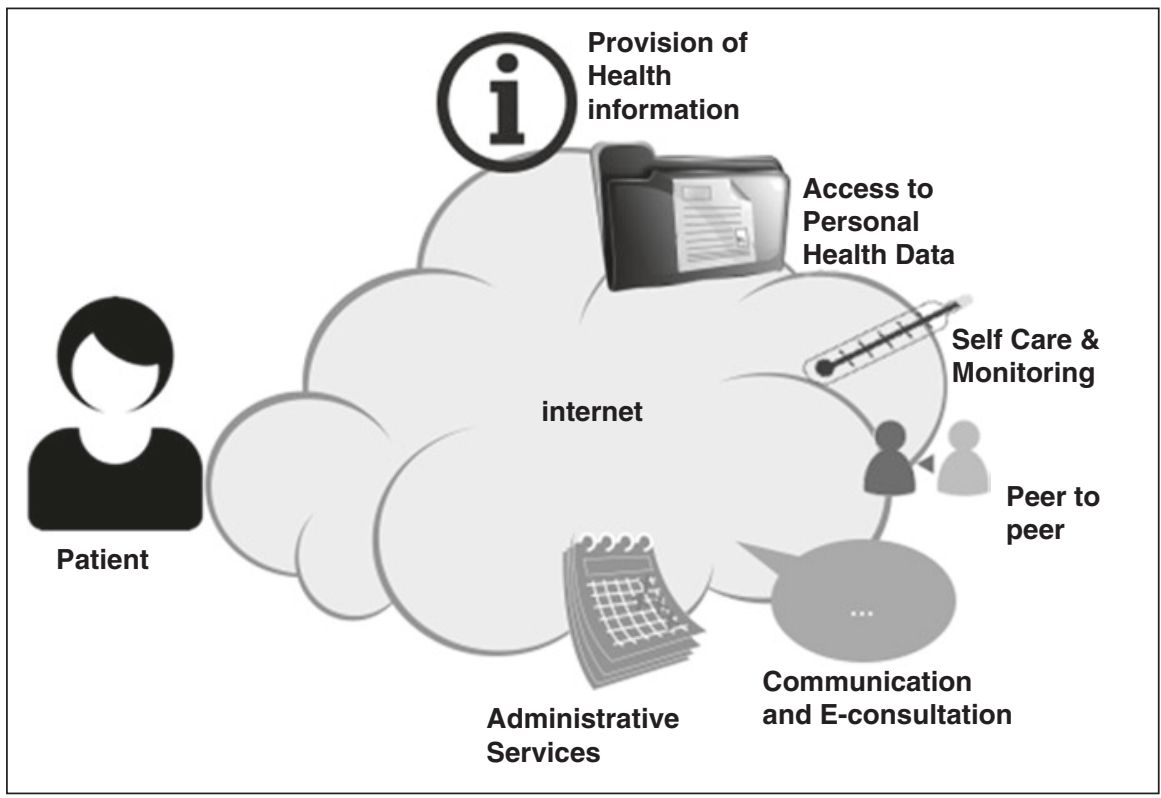

Fig. 2.2 Patient-oriented eHealth services

Additionally, a range of services for self-monitoring and self-care are made available to patients. Some of these services do not entail any involvement of healthcare practitioners while others are linked to healthcare providers that take responsibility for care plans and may assess the information collected. Furthermore, patient-oriented eHealth services may also support peer-to-peer patient networks and forums and in some cases, connections to social media platforms.

Patients and citizens are also offered administrative eHealth services. For instance, many countries offer to patients the possibility to choose among health care service providers, check waiting times, and book appointments. Additionally, solutions for e-consultation services and more generally, electronically supported patient-healthcare provider communications are also in place, often by GP offices in primary care. With these solutions patients are given secure electronic channels for online communication. E-consultation services are mostly used for asking follow up questions after a consultation, asking about medication use and passing on to healthcare providers health related data from self-monitoring practices.

Many European countries have established governmental eHealth patient portals with the aim of offering to citizens one single entry point to the various patientoriented eHealth services offered in the public health sector. 


\subsubsection{Drivers for Patient-Oriented eHealth Projects}

Many health strategies and policies contain visions of more patient-centric healthcare systems (Klecun 2016). Several countries initiated the development of patient-oriented eHealth solutions seeking to realize visions for patient-centeredness. The informed and empowered patient is prominent in the visions. Within medicine, the formulation of "patient-centered care", as articulated nearly a century ago (Peabody 1927) promotes a model of care that entails keeping patients informed, involving them in decisions and self-care management activities, and acknowledging their experience of illness and psychosocial context. In the seminal "Crossing the Quality Chasm" report (Institute of Medicine 2001) patient-centred care was defined as: "providing care that is respectful of and responsive to individual patient preferences, needs, and values, and ensuring that patient values guide all clinical decisions". Patients are seen as integral part of the care team and responsibilities of care-taking and monitoring are partially transferred to patients. Empowerment, transparency and individualization of treatments are emphasized. To realize these visions, new information and communication solutions need to be provided for both patients (enabling them to contribute meaningfully in decision-taking) and providers (providing them better insight on patient circumstances). Such eHealth solutions can support communications, information sharing and distributed data management. Hence, eHealth is seen as a core mechanism for reorienting healthcare towards patient-centeredness.

Another driver for patient-oriented eHealth is a more managerial vision to improve the efficiency of healthcare provision. Organizing shared care solutions around individual patients is expected to help overcome existing communication barriers between institutions and across administrative levels. For instance, a shared patient record system, may help to bridge unconnected "islands" and allow a more efficient overall utilization of resources (Ball et al. 2007; Piras and Zanutto 2010). Furthermore, providing patients with solutions that will allow them to make informed choices can put them in a quasi-customer role. This new patient role is expected to to incentivize a stronger focus on quality and efficiency within the sector. For instance, new patient-oriented services that provide comprehensive information on performance indexes for particular health providers (such as waiting times or treatment-related infection rates) aim to facilitate the patient as a 'customer' to make choices that may create a better working healthcare sector.

Another discourse related to patient-oriented eHealth is the one that emphasizes prevention and the responsibility of each individual to conduct responsible health choices. As such, the scope of attention is expanded from "patients" towards "citizens", i.e. healthy members of the society. This discourse therefore, is not only about disease and treatment, but also, about health and wellness related activities, products, and services that address lifestyle, nutrition and exercise. Currently, information from the mobile applications and devices for self-monitoring used by healthy persons are rarely transferred to the wider institutionalised health system. However, there are initiatives for the provision of eHealth services that can enable the fusion of such privately collected information with medical records. Wellness and health related technologies also enable service models that involve cross-border movement 
and globalization of health service provision. Furthermore, the spread of medical surveillance of patients living at home (including telemedicine solution and welfare technologies) also produces new data streams, with new potentials for analysis and use, and new requirements for infrastructures. Awareness is arising of the need to provide platforms that are able to receive and integrate data of this kind, often coming through "third-party" or non-health related solutions.

Finally, eHealth services may also seek to support peer-to-peer patient networks or more flexibly organized health communities (Eysenbach 2008; Spagnoletti et al. 2015). Peer networks may help patients cope with handling their disease, help navigating the health system or contribute to political work such as awareness and attention to specific patient groups. Based on collecting patient data that are shared in such peer networks, new types of research are becoming now feasible, sometimes organized and coordinated by the patient collectives themselves (Kallinikos and Tempini 2014).

\section{Conclusion}

E-prescription and patient-oriented eHealth services respond to different needs of citizens and healthcare providers and have different roles within European health systems. Overall, e-prescription is more well-defined than patient-oriented services in terms of functionality and in many cases is deeply embedded within pre-existing applications and prescribing tools. Nevertheless, both e-prescription and patient-oriented services have the potential (and frequently the explicit aim) to transform healthcare delivery. E-prescription initiatives are usually seen as opportunities to improve healthcare delivery by systematic and not dramatic change (controlling the ever-increasing medication costs, improving patient safety and providing rich information for performance management). Patientoriented eHealth services are usually seen as opportunities to pursue wider and more radical innovation, aiming to strengthen the patients' role and to facilitate a shift from provider-centred healthcare towards patient-centeredness.

eHealth infrastructures are expected to instigate the reshaping of core roles and relationships within the healthcare systems (Vikkels $\emptyset$ 2010). Therefore, eHealth is not just about more effective 'tools' for addressing particular problems, but needs to be seen as part of longer and more transformative processes of 'digitalization' (Tilson et al. 2010). Digitalization will transform the existing relationships and institutions in healthcare. For example, electronic tools are changing the clinical encounter between a healthcare professional and a patient (May 2007; Winthereik 2008).

Despite having such a transformative orientation, the novel eHealth infrastructures typically leverage existing services, capabilities, institutions, data sources, systems, and communication channels. These sometimes exist within the healthcare providers' organization, and sometimes they can be built upon applications that are not part of the official healthcare system. The eHealth infrastructures can be part of nationally governed initiatives, or initiatives growing out of local action, e.g. from hospitals or health plan providers. 
Expectations are that eHealth infrastructures will help governments improve the quality and efficiency of healthcare and achieve better coordination of care. However, the introduction of novel technologies will not in itself bring into these changes into effect. The underlying premise for this book is the recognition that technology is not an invariant in a transformation process - rather we may expect that any solution will be contested and that it will change shape during realization, implementation and usage. Both technology and institutional transformations trigger complex change processes (Agarwal et al. 2010, Davidson and Chismar 2007) with a reciprocal interaction between technologies and organizations. The stories of building eHealth infrastructures included in this book illustrate several aspects of such complex, interactive transformation processes.

\section{References}

Agarwal R, Gao GG, Desroches C, Jha AK. Research commentary-the digital transformation of healthcare: current status and the road ahead. Inf Syst Res. 2010;21:796-809.

Ball M, Carla Smith N, Bakalar R. Personal health records: empowering consumers. J Healthe Inf Manag. 2007;21:77.

Cornford T, Hibberd R, Barber N. The evaluation of the electronic prescription service in primary care: final report on the findings from the evaluation in early implementer sites. London: University College London; 2014.

Davidson E, Chismar W. The interaction of institutionally triggered and technology-triggered social structure change: an investigation of computerized physician order entry. MIS Q. 2007;31:739-58.

Drucker PF. Management challenges for the 21st century. New York and Oxon: Routledge; 2007.

European Commission. Ministerial Declaration [Online]. Brussels. 2003. Available: http://ec. europa.eu/information_society/eeurope/ehealth/conference/2003/doc/min_dec_22_may_03. pdf.

Eysenbach G. Medicine 2.0: social networking, collaboration, participation, apomediation, and openness. J Med Internet Res. 2008;10:e22.

Hillestad R, Bigelow J, Bower A, Girosi F, Meili R, Scoville R, Taylor R. Can electronic medical record systems transform health care? Potential health benefits, savings, and costs. Health Aff. 2005;24:1103-17.

Institute of Medicine. Committee on Quality of Health Care in America. Crossing the quality chasm: A new health system for the 21st century. Washington: National Academies Press. 2001.

Institute of Medicine. Digital infrastructure for the learning health system: the foundation for continuous improvement in health and health care: workshop series summary. Washington: National Academies Press. 2011.

ISO/TR 20514. Health informatics-Electronic health record-Definition, scope and context. In: Technical Committee ISO/TC 215, H. I. (ed.) ISO. Geneva. 2005.

Kallinikos J, Tempini N. Patient data as medical facts: social media practices as a foundation for medical knowledge creation. Inf Syst Res. 2014;25:817-33.

Kellermann A, Jones S. What it will take to achieve the as-yet-unfulfilled promises of health information technology. Health Aff. 2013;32:63-8.

Kierkegaard P. E-prescription across Europe. Heal Technol. 2013;3:205-19.

Klecun E. Transforming healthcare: policy discourses of IT and patient-centred care. Eur J Inf Syst. 2016;25:64-76. 
May C. The clinical encounter and the problem of context. Sociology. 2007;41:29-45.

OECD. Health statistics (year of reference: 2011) [Online]. 2013. Available: http://www.oecdilibrary.org/social-issues-migration-health/data/oecd-health-statistics_health-data-en.

Peabody FW. The care of the patient. J Am Med Assoc. 1927;88:877-82.

Piras EM, Zanutto A. Prescriptions, x-rays and grocery lists. Designing a Personal Health Record to support (the invisible work of) health information management in the household. Comput Supported Coop Work (CSCW). 2010;19:585-613.

Rodon J, Silva L. Exploring the formation of a healthcare information infrastructure: hierarchy or meshwork? J Assoc Inf Syst. 2015;16:394.

Spagnoletti P, Resca A, Sæb Ø Ø. Design for social media engagement: insights from elderly care assistance. J Strateg Inf Syst. 2015;24:128-45.

Stroetmann K, Artmann J, Stroetmann V, Protti D, Dumortier J, Giest S, Walossek U, Whitehouse D. European countries on their journey towards national eHealth infrastructures. Luxembourg: Office for Official Publications of the European Communities; 2011.

Stroetmann K, Artmann J, Dumortier J, Verhenneman G. United in diversity: legal challenges on the road towards interoperable eHealth solutions in Europe. Eur $\mathrm{J}$ Biomed Inform. 2012;8:3-10.

Tilson D, Lyytinen K, Sorensen C. Digital infrastructures: the missing IS research agenda. Inf Syst Res. 2010;21:748-59.

Vassilakopoulou P, Tsagkas V, Marmaras N. From "rules to interpret" to "rules to follow": ePrescription in Greece. Electron J e-Gov. 2012;10:147-55.

Vikkels $\varnothing$ S. Mobilizing information infrastructure, shaping patient-centred care. Int J Public Sect Manag. 2010;23:340-52.

Winter A, Haux R, Ammenwerth E, Brigl B, Hellrung N, Jahn F. Health information systems: architectures and strategies. Health information systems. London Dordrecht Heidelberg New York: Springer. 2011.

Winthereik B. Shared care and boundaries: lessons from an online maternity record. J Health Organ Manag. 2008;22:416.

World Health Organisation. WHO Global Health Expenditure Database (reference year 2011) [Online]. 2014. Available: http://apps.who.int/nha/database.

World Health Organisation. From innovation to implementation. Copenhagen: WHO Regional Office for Europe; 2016a.

World Health Organisation. Health topics: eHealth [Online]. 2016b. Available: http://www.who. int/topics/ehealth/en/. Accessed 25 June 2016.

Open Access This chapter is distributed under the terms of the Creative Commons AttributionNonCommercial 2.5 International License (http://creativecommons.org/licenses/by-nc/2.5/), which permits any noncommercial use, duplication, adaptation, distribution and reproduction in any medium or format, as long as you give appropriate credit to the original author(s) and the source, provide a link to the Creative Commons license and indicate if changes were made.

The images or other third party material in this chapter are included in the chapter's Creative Commons license, unless indicated otherwise in a credit line to the material. If material is not included in the chapter's Creative Commons license and your intended use is not permitted by statutory regulation or exceeds the permitted use, you will need to obtain permission directly from the copyright holder.

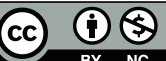

